In a second trial we compared a control diet (maize-soyabean meal) with a diet containing 36 p. 100 peas stored for one year and with two diets containing 36 p. 100 peas of the last crop supplemented or not with tryptophan. Storage of peas did not change its feeding value since the nutritional characteristics remained constant. Supplementation with tryptophan increased the animal performance. During the growing period the feed efficiency of the supplemented diet was improved by 7 p. 100 as compared to the nonsupplemented diet, but remained 11 p. 100 lower than that of the control diet. During the finishing period it was $5 \mathrm{p} .100$ higher than that of the non supplemented diet and was equal to that of the control diet.

\title{
Utilization of spring peas associated with wheat or maize in bacon pig diets
}

\author{
F. GROSJEAN, J. CASTAING * \\ I.T.C.F., 8, avenue du Président-Wilson, 75116 Paris \\ A.G.P.M., 122, boulevard Tourasse, 64000 Pau \\ France
}

The purpose of the present study was to determine the maximum level of incorporation of spring peas into bacon pig diets, while that of winter peas is already well-known (15 p. 100).

In a first trial made on a group of 180 minimal disease Large White pigs, we compared, according to a factorial design, performance of animals receiving 0,15 or 30 p. 100 peas in a simple diet based on maize or wheat supplemented with soyabean meal. In a second trial made on a group of 96 minimal disease Large White pigs we studied the total replacement of soyabean meal by 37 p. 100 spring peas supplemented or not with industrial lysine. We compared these two diets to a control diet without peas and to a diet containing 30 p. 100 peas.

Both trials showed that it was possible to incorporate $30 \mathrm{p} .100$ spring peas into bacon pig diets without reducing the performance over the whole fattening period despite a slight decrease in growth between 25 and $60 \mathrm{~kg}$. Incorporation of peas did not reduce the carcass yield and body composition. Its effect on growth and carcass quality was identical whatever the basal cereal used (maize or wheat).

The second trial showed that total replacement of soyabean meal by peas led to a reduction in the performance which was only very slightly compensated by the lysine supplementation.

\section{Utilization of cereals in simple diets for weaned piglets Comparison of two physical forms : meal and pellets}

\author{
J. FEKETE, J. CASTAING*, O. LAVOREL, M. LEUILLET \\ I.T.C.F., 8, avenue du Président-Wilson, 75116 Paris \\ * A.G.P.M., 122, boulevard Tourasse, 64000 Pau \\ France
}

Six trials were made with 2832 piglets in the same conditions and in two experimental stations with the aim of comparing the performance of piglets fed a cereal-based diet (maize, wheat, barley) offered in two different physical forms : meal or pellets. The incorporation levels of cereals varied from 54 to 64 p. 100 . 
Piglets weaned at the mean age of 26 days $(6.5 \mathrm{~kg})$ were fed ad libitum for two weeks the 1 st-age diet already used before weaning, then for 28 or 35 days (from 9 to $25 \mathrm{~kg}$ live weight) the experimental diets in the form of meal or pellets.

The most remarkable and regular effect of pellets was an improvement of the feed efficiency (4.9 p. 100 reduction in the feed conversion ratio) by reducing the level of voluntary feed intake $(-4$ p. 100$)$ without noticeably affecting the growth rate $(+1.1$ p. 100). The nature of the cereal did not modify this effect which was similar during the first two weeks and during the whole experiment.

This improvement was slightly lower than that obtained in most of the works quoted in the literature. Nevertheless it is interesting from an economic point of view as long as the cost of pellets is 105 p. 100 lower than that of meals.

\title{
Effect of two hypocalcemic diets on the absorption and retention of calcium and phosphorus in the growing pig
}

\author{
A. POINTILLART, Nicole FONTAINE \\ I.N.R.A., Station de Recherches de Nutrition, 78350 Jouy-en-Josas \\ France
}

Three 10-day balance experiments were carried out separately in 4 growing pigs which had already received a low calcium $(0.1 \mathrm{p} .100)$, a high phosphorus $(1.2 \mathrm{p} .100)$ or a control $(0.9$ p. $100 \mathrm{Ca}$ and 0.8 p. $100 \mathrm{P})$ diet for 6 weeks. Additional data concerning bone metabolism or hormonal regulation (PTH and CT) from other experiments are also presented in this report.

Only the calcium deficient diet reduced the growth rate and produced apparent bone disturbances (deformation of snout). Phosphorus absorption was directly correlated with phosphorus intake whereas calcium intake had no influence on $\mathrm{P}$ absorption. The same phenomenon was also observed for calcium. In spite of a temporary (high $\mathrm{P}$ diet) or steady (low $\mathrm{Ca}$ diet) hypocalcemia, no hyperparathyroidism was observed. Low $\mathrm{Ca}$ and high $\mathbf{P}$ diets decreased plasma CT levels. These diets also decreased the phosphorus retention (20.4 p. $100 \pm 2$ and 36.5 p. $100 \pm 2.4$, respectively) compared with that of the control diet (46.3 p. $100 \pm 1.4)$ and produced a high phosphaturia. Relative $\mathrm{Ca}$ absorption did not change whatever the diet (about $50 \mathrm{p}$. 100 of the intake). However, low $\mathrm{Ca}$ diet decreased urinary $\mathrm{Ca}$ excretion. Nephrocalcinosis appeared with the high $\mathrm{P}$ diet. The low Ca diet caused some evidences of hyperosteolysis such as a high urinary hydroxyproine and a higher percentage of bone marrow spaces. However, some of the data concerning. bone mineral content (BMC) are in contrast with the above results. Moreover, in some cases BMC indicated a stimulated bone resorption with the high $\mathrm{P}$ diet. Discrepancies. between the results obtained for different criteria (BMC, plasma minerals and hormones, balances, etc.) are discussed. In conclusion, as for other species excess in phosphorus is not recommended for pigs. 